\title{
DNase-I Footprinting
}

National Cancer Institute

\section{Source}

National Cancer Institute. DNase-I Footprinting. NCI Thesaurus. Code C19898.

A DNA footprinting method which is commonly used for analyzing specific protein-DNA interactions. In this technique, a restriction fragment containing a specific binding site is labeled at one end and digested with DNAsel. The result is a ladder of bands, representing the various sites at which DNase I cuts. The distribution of radioactivity is visualized by autoradiography. 